\title{
Alternative Space Vehicle Launch Systems
}

\author{
Tanay Sharma ${ }^{1}$ and Chris Chatwin. ${ }^{2}$ \\ University of Sussex, Brighton, East Sussex,BN1 9QT \\ Christopher Long ${ }^{3}$ \\ University of Sussex, Brighton, East Sussex, BN1 9QT, United Kingdom \\ Rupert Young ${ }^{4}$ \\ University of Sussex, Brighton, East Sussex, BN1 9QT, United Kingdom \\ and \\ Phil Birch ${ }^{5}$ \\ University of Sussex, Brighton, East Sussex, BN1 9QT
}

\begin{abstract}
Over the last century chemical propellants have been the dominant fuel source for the aviation and aerospace industry, primarily because they are able to provide ample power for a limited cost. However, over the last decade as the price of chemical propellant continues to soar and their detrimental effect on our environment comes to light, there has been a surge to develop an alternative propulsion system. This has driven us in the search for a greener technology, which is capable of providing similar power without compromising on safety and reliability. This paper proposes the use of magnetic levitation and propulsion within a vacuum chamber as an alternative technology. Since such a system relies on an external energy source, the amount of power that can be supplied to the track and craft is only limited by available technology and power sources. Although magnetic levitation has been used for high speed ground transport before, we shall break from tradition by trying to propose the development of an alternative propulsion system that could be used for commercial aircrafts as well as future space travel.
\end{abstract}

\section{Introduction}

$\mathrm{T}$ HE fantasy of traveling to space can be dated as far back as the second century, when the Greek rhetorician Lucian wrote an account of a voyage to the moon. As time passed the fascination of what lies beyond the skies intensified, and as early as 1869 author Edward Everett Hale depicted a manned satellite functioning as navigational aide to ships in his book The Brick. In 1928 Herman Potocnik laid out detailed plans for a wheel-like space station in his book The Problem of Space Travel, however it was not until 1952 that Wernher von Braun wedded fantasy to physics, and announced his vision of how then-existing technology could be used to put a permanent space station into orbit around the Earth in a groundbreaking article in Collier's magazine ${ }^{1}$. He proposed a 250 foot wide inflated wheel, made from reinforced nylon that would function as a navigational aid, meteorological station, military platform and way station for space exploration. Von Braun's space station was shaped like a wheel with two spokes, which would spin in order to create centrifugal force that would act as false gravity. The station would have consisted of communications equipment, earth observatories, weather forecasting centers, navigational equipment and living space.

In 1968 Stanley Kubrick introduced the masses to 2001: A Space Odyssey a science fiction film that dealt with the thematic elements of human evolution, artificial intelligence and extraterrestrial life. The film sparked the

\footnotetext{
${ }^{1}$ Research Student, Dept. of Engineering \& Design, University of Sussex, Student Member.

${ }^{2}$ Head of Research, Dept. of Engineering \& Design, University of Sussex.

${ }^{3}$ Reader in Mechanical Engineering, Dept. of Engineering \& Design, University of Sussex.

${ }^{4}$ Head of Department, Dept. of Engineering \& Design, University of Sussex.

${ }^{5}$ Lecturer in Engineering, Dept. of Engineering \& Design, University of Sussex.
} 
imagination of a generation and for the first time providing a realistic image of what our future in space might look like. Kubrik's space station had considerable similarities to Von Braun's design and accurately portrayed the contrast between artificial gravity generated by the rotating wheel to the weightlessness experienced outside. In the movie the station had a diameter of 900 feet and orbited 200 miles above the earth, housing an international contingent of scientists, passengers and bureaucrats. The spoke design shown in the movie was a popular concept at the time. The shape combined with the revolution speed created simulated gravity in space. The more scientists learned, however, the more they became aware of the physical hazards and the costs necessary to avoid those hazards. Since the consequences of extended exposure to weightlessness are undesirable physiological adaptations that increase the difficulty of returning to an environment with gravity, creation of artificial gravity was incorporated in various designs. However, studies proved that people get motion sickness in centrifuges with a small rotational radius or with a rotation rate above 2 rotations per minute. As a result, researchers realized that in order to create artificial gravity similar to earths and turn at a speed slow enough to not trigger motion sickness the diameter of the wheel required would be quite large. In effect to generate a rate of spin of 2rpm or less, and produce a gravitational force equivalent to the surface of the earth, the radius of rotation would have to be 224 meters $(735 \mathrm{ft}$ ) or greater. Similarly a rate of spin of 1rpm would virtually eliminate motion sickness, would require a radius of rotation of roughly 894 meters (2933 feet). The design difficulties and extremely high cost of construction meant that the idea of generating artificial gravity via a rotational ring, were dropped while modeling the International Space Station.

To construct the International Space Station, all the material required was transported into space at great expense from the earth's relatively high gravity surface. The design and construction of future deep space habitats is now effectively dependent on progress in three main areas:

1) Development of alternative propulsion technologies, capable of carrying larger payloads at reduced costs

2) Establishment of permanent bases on lower gravity moons which can provide materials that can be transported cheaply to future space habitat orbits.

3) New technology capable of processing lunar materials into useable products at low costs.

In order to achieve permanent establishment on low gravity moons, further research is required into the development of crafts capable of achieving such orbits. It is also vital that these future space vehicles are capable of carrying a much larger payload than the space shuttle and are designed for long term missions. In order to do so, the propulsion technology used for these crafts needs to change radically. By using current day solid fuels our return to the moon is highly unlikely.

Section II discusses the cost and overall effectiveness of the Space Transport System (STS) program, further sections will deal with launching space vehicles using a new technology that combines the use of magnetic levitation and vacuum tubes. This is derived by the implementation of the spacecraft when evaluating the launch of Ithaca - a concept proposal for the first independent commercial space habitat. The crafts reusability and capability of multiple, weather independent launches, allows us to project the assembly of Ithaca ${ }^{2}$ within a five year time span with lower monetary requirements than the ISS.

\section{STS Program Effectiveness}

Born in 1968 at the height of the Apollo program, the Shuttle was designed to provide NASA with an efficient, reusable method of carrying astronauts to and from a large, permanently manned space station ${ }^{3,4}$. It would also function as a multipurpose satellite launch system with the potential to replace the Delta and Titan rockets. Originally slated to enter service in 1977, the Shuttle made its first spaceflight in 1981 following a series of design changes. Initially designed as a two-stage system, recession within the United States and payload demands made by the U.S Air Force forced NASA dramatically altered the design requirements of the Shuttle and proposed space station. This meant that from a Skylab like single structure, NASA switched to a modular concept, deciding that the space station be built over a period of several years. This redesign not only allowed NASA to spread the financial outlay over a longer period but also allowed it to carry heavier military payloads. The mission of the STS program was to operate a high flight rate, at low cost, and with high reliability. It was intended to improve on the previous generation of single-use manned and unmanned vehicles. In order to achieve this goal, NASA initially estimated 2560 missions per year, however by the mid-1980s this had been geared towards a more modest launch rate of 24 . By 1988, following the Challenger disaster, NASA adopted a more relaxed pace averaging about eight launches per year. 
Whereas the Shuttle has been a reasonably successful launch vehicle, it has been unable to meet its goal of radically reducing flight launch costs, as the average cost per launch today is roughly $\$ 450$ million. Although initial launch expenditure projections were between $\$ 10$ and $\$ 20$ million, as of early 2005 the average launch expenditure for Shuttle operations had accumulated to $\$ 1.3$ billion, whereas the overall cost of the STS program amounted to approximately $\$ 24$ billion. In order to derive costs related to the construction of the ISS, expenses for non-ISS flights amounting to $\$ 5$ billion should be subtracted. NASA projected another $\$ 20.5$ billion in shuttle program costs during 2006-2011, while anticipating an average cost of $\$ 2.5$ billion during 2011-2016. It is estimated that by the retirement of the Shuttle the STS program would have cost $\$ 174$ billion, whereas the ISS related costs would be in excess of $\$ 100$ billion - as opposed to media projections of the ISS program costing under $\$ 100$ billion. One of the primary reasons for soaring launch costs is the high-cost maintenance schedule of the STS program. When originally conceived, the Shuttle was to operate similarly to an airliner, and be ready for launch in as little as two weeks. However, the turnaround time for the Shuttle can now be up to a few months. This is mainly due to stringent documentation requirements, brought about in the wake of the Challenger and Columbia disasters. This paperwork results from the fact that unlike current expendable vehicles, the Space Shuttle is manned and has no escape systems mode for most of the flight regime. As a result an incident resulting in the loss of a booster would also result in the loss of the crew. Furthermore, there are cases where there are no abort modes; as a result each piece of hardware must be checked to ensure that

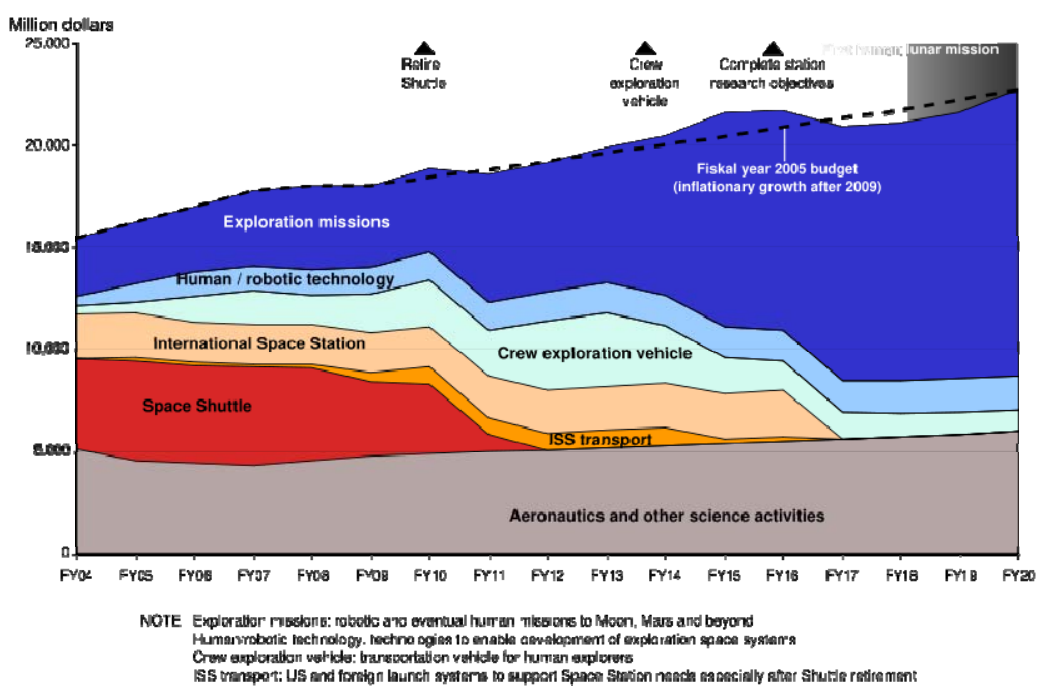

Figure 1. NASA Main Budget Projections for FY 2004 to FY 2020 it functions perfectly. This results in a massively inflated labor cost, with around 25,000 workers in Shuttle operations and labor costs of about \$1 billion per year.

\section{Magnetic Levitation \& Propulsion (MagLev)}

The idea of using magnets to achieve high speed transport systems using non-contact magnetically levitated vehicles was proposed in the early nineteen hundreds. However, limited by available technology, no practical method could be devised to achieve this goal. In 1934 Hermann Kemper, a German scientist, received a patent for the idea of using magnetic levitation and propulsion to run high-speed ground transport systems. His development fuelled extensive research in the use of magnetic levitation technology in other countries, leading to the development of a few large scale models. Although the current use of this technology is restricted to high-speed ground transport systems, its application for orbital transfer have been studied extensively due to its energy efficiency, environmental benefits and high-velocity transport capabilities. The efficiency of such a system in terms of kilowatt hours per tonmile for freight is much lower than that for commercial airplanes. It is virtually pollution free, and allows the use of renewable sources of energy, thereby not contributing to the ever growing problem of global warming. It is weather independent, and can carry enormous traffic loads of both people and cargo on a narrow guideway. Whilst the average cost per Lb to orbit for current day LEO systems ranges between \$3632-\$4587, and that for GTO systems ranges from $\$ 9243-\$ 11243^{5}$, MagLev technology is capable of providing higher payload delivery at a fraction of the cost. A system designed on the MagLev principle requires horizontal launch, which in turn ensures the safety and reliability of the vehicle, as vehicle recovery and emergency launch aborts are easier to carry out. In such a system the intended vehicle is suspended, guided and propelled using electromagnetic force, thereby eliminating the need for chemical propellants. Also as there is no physical contact between the vehicle and the guideway the maintenance cost of the guideway is considerably reduced ${ }^{6,7}$.

Since MagLev technology cannot share existing infrastructure, it needs to be designed as a complete system. In order to do so, it is important to calculate the maximum load the guideway is required to support before the design stage, as making changes later on can prove to be an expensive process. An important goal for a MagLev system 
design is the reduced cost per load factor, so that it is economically viable and capable of competing with current propulsion technologies. Of the various MagLev possibilities, systems based on superconducting magnets best achieve this goal, as they act as perfect diamagnets and completely expel magnetic fields due to the Meissner effect ${ }^{6}$. The levitation in such magnets is stabilized by flux pinning within the superconductor. Today MagLev technology is successfully used in high-speed ground transportation and utilizes one of the following methods:

4) Electromagnetic Suspension (EMS): The vehicle levitates above a steel guideway Whereas electromagnets attached to it are oriented toward the guideway from below. The electromagnets use feedback control to maintain a vehicle at a constant distance from the track. Magnetic fields inside and outside the vehicle are insignificant. EMS is a commercially available technology that is capable of attaining high speeds and does not require a secondary propulsion mechanism. However, the separation distance between the levitated vehicle and the guideway needs to be constantly monitored and corrected to avoid collision due to the unstable nature of electromagnetic attraction. Also due to the systems instability, constant corrections by external sources bring about vibration issues.

5) Electrodynamic Suspension (EDS): In this system both the guideway and the vehicle exert a magnetic field, and the repulsive force between these magnetic fields levitates the vehicle. The magnetic field in the vehicle is produced either by an array of permanent magnets or by superconducting electromagnets, whilst the repulsive force in the guideway is created by an induced magnetic field in internal wires. The large margin between the vehicle and the guideway allows for high speeds; however strong magnetic fields onboard prohibit the use of magnetic data storage devices. As a result the vehicle used in such a system may require magnetic shielding. Also in such a system, the vehicle would initially require an alternate propulsion source as the current induced and the resultant magnetic flux is not large enough to support the vehicles weight.

6) Magnetodynamic Suspension (MDS): In this system no power is required to activate the magnets within the vehicle. The separation between the vehicle and the guideway is automatic and requires no outside correctional control. Since the attractive force of permanent magnets is far greater than the repulsive force, smaller, cheaper magnets can be used for the system. Magnetic fields, both inside and outside the vehicle are insignificant and in case of a power failure the vehicle slows down on its own safely. Like EDS, this system also requires an alternate source for propulsion at low speeds, which allow the vehicle to overcome the electrodynamic drag it faces. Once the vehicle's velocity increases, there is a net increase in the levitation force allowing the system to support the vehicles weight. Due to the nature of this set-up, all aspects are calculated and designed prior to construction, leaving little room for adjustment after fabrication. However, due to the use of steel and permanent magnets there is no limitation on the speed the vehicle may attain while it is suspended.

\section{Proposed Propulsion Mechanism}

An open air Maglev system is subject to both electrodynamic and aerodynamic drag. While the electrodynamic drag is negligible at high speeds, the aerodynamic drag faced quadruples every time the crafts velocity doubles. Therefore the power required to overcome the drag is eight times the original value for effective increase in velocity. The frictional drag faced by the vehicle during a horizontal launch would also lead to a large increase in the surface temperature at the vehicles extremities, which may damage various internal components. As a result the maximum speed of current day ground transport systems operating on MagLev principle is capped at 350mph. The aerodynamic drag faced by such a system is proportional to the air density, hence if we were to reduce the air density coming in contact with the launch vehicle, we would effectively lower the amount of power required to overcome the drag force. In order to overcome the drag and create a reliable and economically viable system, we propose the use of superconducting MagLev technology inside a purpose built vacuum tunnel approximately five miles long, allowing us to provide initial launch velocity to a fully reusable launch vehicle (RLV). The vacuum tube has an air handling and control system that allows the tube ambient pressure to be equalized with the ambient air pressure just as the space vehicle exits the launch tube, the system is described in section VIII of this paper.

This horizontal launch approach within the confines of a tunnel, allows the vehicle to attain much higher speeds by minimizing the negative impact of aerodynamic drag. The electrodynamic drag the vehicle would face at low speeds can be controlled using a null flux suspension mechanism, which reduces the power losses in the guideway due to induced current.

Such a system can be designed in two distinctive ways:

1) A vehicle based design - by using such a method the vehicle is levitated directly over the guideway, and is propelled magnetically using a series of linear synchronous motors (LSM). Within the LSM, small 
alternating current magnets push on superconducting magnets to provide a net propulsive force. Whereas the magnets in the LSM are AC magnets those on the vehicle are DC, allowing the magnetic polarity to alternate along the vehicle.

2) Mass driver design - by using such a method the vehicle is placed on a purpose built magnetisable stage, which is then levitated and accelerated by the sequential firing of a row of electromagnets. Once the vehicle is accelerated to optimum speed the two separate and the stage is slowed down and recycled for another launch. After leaving the guideway the vehicle continues to move due to inertia.

The key issue with the mass driver design is that it is only practical for accelerating small objects ${ }^{8,9}$. The limitations on the design are imposed primarily by the cost of the silicon to switch current and the cost of the power supply and temporary storage. However, based on such a design if a vehicle weighing approximately 1.5 tonnes was to be accelerated at a speed of $20 \mathrm{~km} / \mathrm{s}$ (at sea level), it would traverse the $8 \mathrm{~km}$ atmosphere in half a second emerging at a speed of $16 \mathrm{~km} / \mathrm{s}$, enough to escape the solar system. Although the launch energy required for such a system seems extremely large, it amounts to roughly 83MW-hrs which corresponds to only a few minutes output of any major metropolitan utility plant. Also whereas the length of an effective launcher would exceed 12.5 miles for $1000 \mathrm{~g}$ acceleration, by providing an attainable $10,000 \mathrm{~g}$ acceleration we can reduce the length to 1.25 miles. It is essential to remember that although energized by capacitors, the costliest and bulkiest energy source known, each capacitor is fired over a hundred times during each launch cycle by being connected to multiple drive coils.

\section{Launch System Location}

Once a system design is chosen, the next key step is choosing a construction location. Since the system would be primarily designed for space launch rather than commercial use, it would be more effective closer to the equator as the thrust and fuel requirements for sending a vehicle into orbit are lower due to the earth's rotational speed. Also, being closer to the equator the earth's rotational speed provides an added boost to the vehicle's velocity (usually around 6\%). As such, current day systems launched from Cape Canaveral in Florida gain an approximate boost of $911 \mathrm{mph}$ whereas those launched from the French Guinia (5 degrees from the equator) gain roughly a 1000mph boost $^{2}$. The active magnetic guidance and flexibility in design of the propulsion system allow the guideway to be adapted to various landscape conditions. Current MagLev systems such as the Transrapid have successfully proved that they can operate at full speed $(280 \mathrm{mph})$ at a gradient of 10 degrees $^{10}$. Bearing that in mind since the proposed guideway would be enclosed and the operational speeds much greater than $280 \mathrm{mph}$, a sudden gradient of 10 degrees would cause massive g-forces to accumulate. As a result, ideally the system should be built on the side of a mountain as that would provide the launch system with gradual inclination. The system could also be constructed on a flat plain, but by doing so it would require a larger power input.

Based on these considerations, mount. Kilimanjaro in north-eastern Tanzania was chosen as the launch location. At latitude of $3^{\circ}$ it lies closer to the equator than the Kourou Launch Station used by the European Space Agency, and hence fewer changes are required for launch trajectory. Launches carried out at this facility would also benefit from the above mentioned slingshot effect, which would provide the launch vehicle a $1000 \mathrm{mph}$ boost $^{2}$. Other than the physical characteristics that appeal to placing the project in Tanzania, it is an ideal country for investment. It has a relatively stable political situation and its economy has progressed steadily since the implementation of the structural reform program in the mid 1990's. Furthermore, whereas initiating the project in a developed country would increase labor and manufacturing costs, developing nations offer not only lower costs of living but also a

large work forces at reduced costs. Since such a launch system would be constructed as part of an international alliance, it would provide the local environment with significant economic benefits. Not only would the project inject an estimated \$118.850 million in the local economy over two years, it would also help in the overall development of Tanzania.

\section{Launch System \& Vehicle Design}

Since the proposed launch system requires various technologies to work in harmony, each of them must be designed to meet specific requirements. As a result the three key areas during the design phase are:

1) Tunnel: As mentioned earlier the tunnel required for the proposed system would be approximately five miles long. Not only will this tunnel house the guideway, it would also have an intricate network of gas pipes and electrical cabling. Whereas construction seems relatively easy, it is important to remember that the tunnel would also be subject to shockwaves during launch vehicle acceleration. It should not only be able to withstand these shockwaves but must also provide adequate protection to the gas pipes housed within it, as a ruptured pipe could cause a catastrophe. Furthermore, the tunnel must also provide the system housed within it adequate protection from the elements. Since the launch system is intended to have a shelf life of over 
2) Guideway: The guideway housed within the tunnel is used to propel the launch vehicle to orbital velocity using MagLev technology. In order to design an efficient system the five mile guideway is split into multiple sections. Each section of the guideway would be powered by an external source, and activation of the various sections controlled by a central system. As such, only single sections of guideway would be active at any given time, as illustrated in Fig. 2. Once the launch vehicle clears a section of guideway, power to that section is automatically cut. With the speeds the launch vehicle is designed to attain, it is imperative to ensure that the guideway control system is able to provide adequate power in fractional time intervals.

3) High-Pressure chambers and piping network: The high pressure chambers and

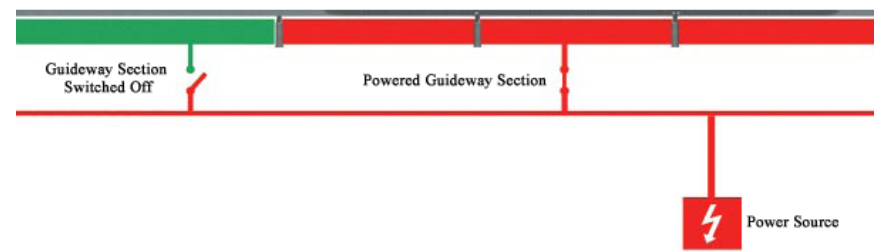
piping networks are designed to extract air from the tunnel before vehicle acceleration begins. The extracted air is then pressurized and returned via the same piping network to large valves placed throughout the length of the tunnel. Each valve is attached to 150 converging nozzles which are manufactured to operate at design conditions. A gas control system ensures that the nozzles remain in a choked condition permitting accurate air flow control. When instructed, the gas control system regulates the pressure such that all the convergent nozzles operate simultaneously, allowing sonic gas flow into the tunnel. The number of nozzles required to fill the tunnel depend not only on the time constraints but also on the mass flow of each nozzle and the net volume of the tunnel. The designed system should incorporate switchable extra inactive nozzles, which could be used in a contingency situation.

The main consideration while designing the launch vehicle was to allow the vehicle to carry a larger payload than the Space Shuttle. By eliminating the need for carrying oxidizers, the proposed launch vehicle is already $104,000 \mathrm{~kg}$ lighter than the Shuttle orbiter at the time of launch. Furthermore, the launch vehicle would not only possess a MagLev capable base but would also have two scramjet engines. Despite its size, in order to land safely the vehicle is designed to be as agile as possible, in order to shake off excess speed at re-entry. The preferred method of vehicle recovery would be a horizontal landing, which can be achieved on any conventional airstrip. In order to effectively use the two scramjet engines, the launch vehicle is designed using the waverider principle as illustrated in Fig. 1. Due to this design principle the launch vehicle effectively requires only half a scramjet engine. The shockwave of the vehicle itself compresses the inlet gasses, forming the first half of the engine. This greatly reduces craft mass and construction effort. Also since scramjets have few or no moving parts, their body consists of continuous surfaces. With simple fuel pumps, reduced number of components and the re-entry system being the craft itself, designing the scramjet engine is reduced to a materials and

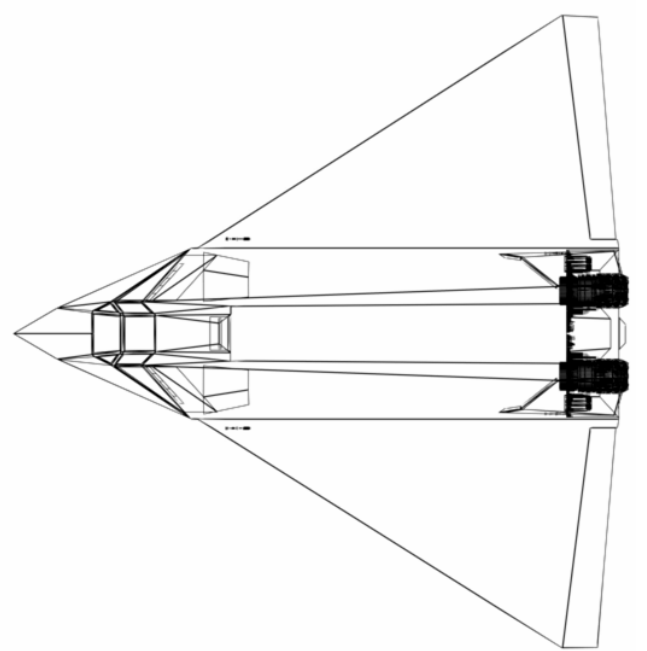

Figure 3. Proposed Launch Vehicle Design modeling problem. Due to the hypersonic speed of the launch vehicle, heat insulation from atmospheric friction would be required throughout the craft. Therefore the proposed launch vehicle would not only be protected by insulation shields similar to the Space Shuttle, but would also employ active cooling, circulating coolant throughout the vehicle skin to prevent it from disintegrating.

\section{Launch System Development}

Once the tunnel and guideway systems are constructed, and work on various support installations is finished, the launch system would be ready for use. From the time of completion, six months would be devoted to the 
development of launch schedules and rigorous testing of all control systems. The overall budget request for launch system development includes the initial cost of launch vehicle research and development; and the project costs for 25 missions, required to complete the Ithaca space station. The budget for the initial manufacturing and testing of the launch vehicle is estimated at $\$ 600$ million. Of this, $\$ 500$ million is manufacturing cost whilst the remainder is used for test flights. The vehicle launch is a multi stage program, divided into development and operational procedures.

Development procedures include preparation programs and support features, which provide contingency planning to assure transportation and assembly support to the Ithaca Space Station. For each mission, a performance monitoring system is employed, which ensures the best possible performance of the craft's engines and overall systems. The monitoring system is a real time control network, which aims to improve engine anomaly response capabilities and overall monitoring of the launch vehicle. Furthermore, the launch vehicle incorporates a flight management control system, responsible for relating critical flight information such as vehicle trajectory and surface temperatures in real time to the ground control center. It is also capable of isolating potential system failures and relaying data to the vehicle monitoring system.

The operational procedures for the launch system can be divided into three main areas:

1) Program Integration: This procedure assures successful technical integration of all the launch vehicle and its payload for each mission, based on the mission requirements. The program integration budget includes funds for analysis, management, reliability and quality assurance functions performed for each mission. Furthermore, it includes the necessary mechanical and avionic engineering tasks to ensure that the vehicle is launched safely and is capable of delivering its payload before a successful return.

2) Ground Operations: This procedure provides final integration and checkout for all hardware elements related to vehicle launch. This includes guideway and vehicle maintenance, coordination and tracking of payload and operations infrastructure supporting launch and recovery procedures for all missions.

3) Flight hardware and software: This procedure ensures that all hardware and software designed for the launch vehicle is developed, manufactured and tested sufficiently before initial launch. It ensures the overall reliability of the launch vehicle, and is vital to the success of each mission. The software activities included in the flight hardware budget incorporate the development, formulation and the verification of the guidance and navigational system software for the launch vehicle.

\section{Vehicle Launch Phases}

After integration of the launch vehicle and its payload, the following three stages are observed for successful orbital launch:

1) Depressurization: Once the launch vehicle is in place over the guideway, multiple extractor jets are activated to reduce the density of air within the tunnel. These jets deliver the expelled air into large high pressure storage tanks placed throughout the system. Once the pressure within the tunnel is reduced to nominally zero, the first section of the guideway is activated. This process also automatically activates the magnets on the undercarriage of the launch vehicle. After initial checks are completed, the landing gear of the launch vehicle is retracted. At this point the vehicle is automatically controlled and leveled using onboard computers. The control systems for the guideway ensures that it is capable of supporting the weight of the launch vehicle, and automatically determines the power required throughout the guideway for a successful launch. They also set activation times for the remaining sections of the track. Once this information is relayed to the control center and the vehicles onboard computers the craft begins acceleration.

2) Acceleration: as the launch vehicle begins to accelerate, guideway control systems use data acquired from the vehicles onboard computers to increase the overall speed of the craft, ensuring that it is capable of reaching orbital velocity before reaching the end of the guideway. During acceleration, communication loss between the vehicles computers and the guideway control systems leads to an automatic abort. In such a scenario, the power output to the active section of guideway is reduced and the vehicles landing gear is engaged.

3) Pressurization: even before the launch vehicle begins to accelerate, the storage tanks placed throughout the system deliver pressurized air to over two hundred thousand converging choked nozzles. The gas pressure is maintained by an automated system which ensures that the nozzles remain choked until the ambient atmospheric pressure is reached. As the launch vehicle approaches the last section of the guideway, the tunnel pressure has increased from zero to ambient atmospheric pressure. This ensures that a standardized pressure exists both inside and outside the launch tunnel. 
It is important to remember that since the proposed launch vehicle is entirely reusable, the same vehicle is capable of providing multiple launches each month. If a fleet of launch vehicles was to be designed, we could theoretically launch multiple times each day. Once Ithaca is completed, the proposed launch system could be expanded to incorporate a spaceport, whilst the launch vehicle provides regular orbital flights to paying customers. This would make the proposed system unique, as it would be the first system capable of transporting passengers, scientific cargo and military equipment into orbit. Further modifications to the launch vehicle would allow it to use Ithaca as a fueling base for deep space exploration.

\section{Ithaca Space Station}

The Ithaca space station is intended to be the first commercial space station, which would act as a permanent metrological station, navigational aid and a hub for deep space exploration ${ }^{2}$. It is designed to accommodate the needs of both researchers and space tourists, and would have a capacity of a thousand inhabitants. Ithaca was primarily proposed to test the cost effectiveness of the alternative launch system and launch vehicle described earlier. The station is based on a standard torus design, and is projected to have a rate of rotation of 1.4rpm, which would be enough to generate artificial gravity. At its core, the structure would house an anti-gravity experiment facility, which would be designed to conduct experiments taken over from the current ISS. A basic overview of the station is presented in Fig 4.

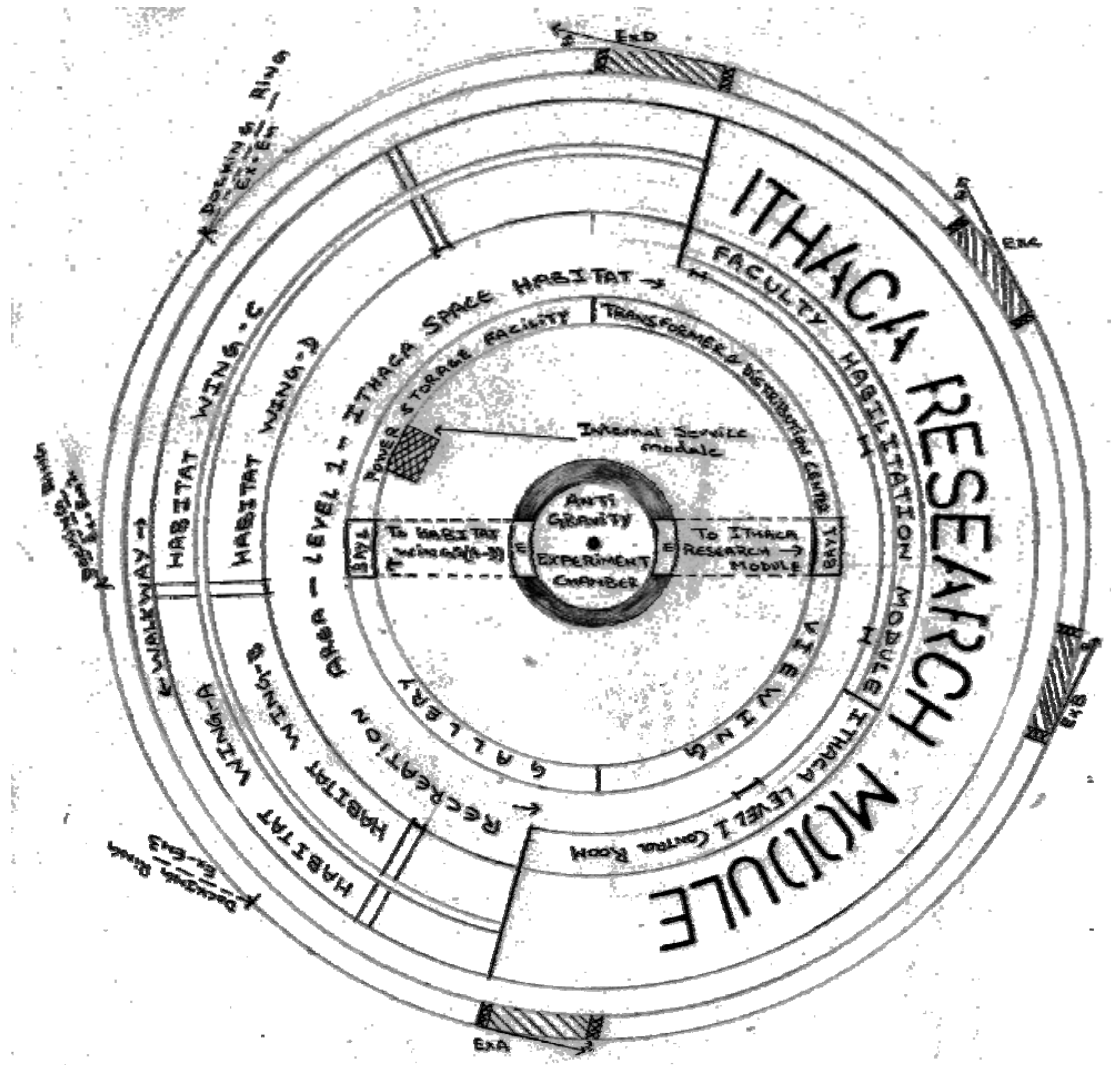

Figure 4. ITHACA Space Station Proposal

\begin{tabular}{|c|c|c|}
\hline \multicolumn{3}{|c|}{ Ithaca Station Station } \\
\hline \multicolumn{3}{|c|}{ Station Requirements } \\
\hline Population & & 1000 \\
\hline Major Radius & $(\mathrm{m})$ & 450 \\
\hline Minor Radius & $(\mathrm{m})$ & 65 \\
\hline Gravity & (g) & \\
\hline Air Pressure & Katt & \\
\hline \multicolumn{3}{|c|}{ Calculated Values } \\
\hline Area/Person & $(m 2)$ & 246.0956 \\
\hline Surface Area & $(m 2)$ & 7469048.8 \\
\hline Volume & $(\mathrm{m} 3)$ & $2.50 \mathrm{E}+07$ \\
\hline Shielding & (Mt) & 3.537 \\
\hline Oxygen Mass & (kt) & 9.185 \\
\hline Nitrogen Mass & (kt) & 36.741 \\
\hline Structural Mass & (kt) & 163.807 \\
\hline Rate of rotation & (rpm) & 1.4088 \\
\hline
\end{tabular}

Table 1. Station Properties

The various specifications for the Ithaca space station are detailed in Table 1. Although various materials were investigated for the construction of the space station, keeping the overall mass of the station as low as possible was essential; since structural weight reduction is vital for maintaining low flight costs. As a result materials with low density are most appealing. Hence Al-Li alloys were considered, as they have lower density than similar alloys, have excellent fatigue and cryogenic toughness properties and superior fatigue crack growth resistance ${ }^{2}$. The physical properties of Al-Li alloy 8090 chosen for construction are detailed in Table 2. 
The Space Station would be manufactured in sections, and be transferred to via separate missions due to payload specifications. It would be assembled and placed in a sun-synchronous orbit $700 \mathrm{~km}$ from the earth's surface and would have a $45^{\circ}$ inclination so that it has continual exposure to the sun. For the assembly and transportation of the Ithaca space station, the proposed launch system and vehicle would be used. It is estimated that a total of 25 missions would be required to deploy the main structure of the space station and another 20 missions required to complete construction. As a result the projected overall cost per manned mission is estimated at $\$ 344.145$ million, which is considerably lower than the current Shuttle missions. By using a reusable launch vehicle, we ensure

\begin{tabular}{|c|c|}
\hline Property & 8090 \\
\hline Density, g/cm ${ }^{3}$ & 2.55 \\
\hline Melting range, $\mathrm{C}$ & $\begin{array}{l}600- \\
655\end{array}$ \\
\hline Elastic modulus, GPa & 77 \\
\hline $\begin{array}{l}\text { Thermal conductivity at } 25 \mathrm{C} \text {, } \\
\text { W/m-k }\end{array}$ & 93.5 \\
\hline $\begin{array}{c}0 \\
\text { Specific heat at } 100 \mathrm{C}, \mathrm{J} / \mathrm{kg}-\mathrm{k}\end{array}$ & 930 \\
\hline
\end{tabular}

Table 2. Al-Li Alloy Properties that despite economical fluctuations the financial impact to the launch system is minimal. Since Ithaca is designed to act as a destination for space tourists, substantial operational costs for the station can be recovered by operating chartered flights, and providing docking privileges. Most importantly, Ithaca would act as an ideal platform to conduct deep space human exploration and achieve a better understanding of the known universe.

\section{Conclusion}

With the era of space tourism getting under way, and the imminent retirement of the space shuttle in 2010, ambitious plans for commercial spaceports are beginning to take shape in the United States and around the world. While industry optimists insist that, despite the current economic situation, growth in the commercial space sector is inevitable, there is concern that the market for space travel may not be large enough to sustain multiple spaceports. Based on the current economic climate and the every increasing price of crude oil, future systems must prove their cost effectiveness before obtaining government funding. In order to do so, they must be able to provide a high payload capacity at reduced costs which can only be achieved by the use of alternative fuels. The launch system and craft proposed in this paper intend not only meet those objectives but also provide a greener solution to future space travel. Due to the nature of the system, it can operate in extreme weather conditions and can be adapted to carry both commercial and military payloads. Furthermore, the development of Ithaca would also enable the proposed system to ferry space tourists to the new habitat, generating enough revenue to sustain itself.

Whilst the initial cost of construction may seem extravagant, it is important to remember that the system has minimal maintenance costs and the overall cost per flight, and cost per manned flight, would be significantly cheaper than the current alternatives.

\section{References}

${ }^{1}$ Barry, P., "Wheels in the Sky” Science@NASA [online edition],

URL: http://www.science.nasa.gov/headlines/y2000/ast26may 1m.htm [cited 27 May 2008]

${ }^{2}$ Theodoulou M, "Low Cost Space Vehicle Launch and Propulsion Systems” MSc. Dissertation, Dept. of Engineering \& Design, University of Sussex, Brighton, UK, 2007

${ }^{3}$ Heppenheimer, T.A., “The Space Shuttle Decision: NASA's Search for a Reusable Space Vehicle” Washington D.C, National Aeronautics and Space Administration, 1999,

URL: http://history.nasa.gov/SP-4221/sp4221.htm [cited 12 May 2008]

${ }^{4}$ NASA Space Shuttle News Reference, 1981[PDF Document],

URL: http://ntrs.nasa.gov/archive/nasa/casi.ntrs.nasa.gov/19810022734_1981022734.pdf [cited 18 March 2008]

${ }^{5}$ Sietzen, F. Jr., "Faltering: The myth of \$10,000, per pound,” Spaceref - Space news as it happens [online database],

URL: http://www.spaceref.com/news/viewnews.html?id=301 [cited 12 November 2007]

${ }^{6}$ Moon, F.C., Superconducting Levitation Applications to Bearings and Magnetic Transportation. Wiley, New York 1994

${ }^{7}$ Heller, A., "A New Approach for Magnetically Levitating Trains—and Rockets", Science \& Technology Review, June 1998. URL: https://www.llnl.gov/str/Post.html [cited 15 March 2008]

${ }^{8}$ O’Neill, G.K., Kolm, H.H., “Mass Driver for Lunar Transport as a Reaction Engine,” Jour. Of Astronomical Sciences, Vol. 15, No. 4, Jan-Mar 1976.

${ }^{9}$ Arnold, W., Bowen, S., Cohen, S., Fine, K., Kaplan, D., Newman, J., et al, “Mass Drivers,” part I,II\&III, NASA-AMES Summer Study: “Space Resources and Space Settlements” NASA SP-428,1979,U.S. Govt. Printing Office 